\title{
RELIABILITY OF THE REACTIVE STRENGTH INDEX AND TIME TO STABILIZATION DURING DEPTH JUMPS
}

\author{
Flanagan, E.P., Ebben, W.P.* and Jensen, R.L.** \\ Biomechanics Research Unit, University of Limerick, Limerick, Ireland \\ *Strength and Conditioning Research Laboratory, Marquette University, \\ Milwaukee, WI, USA \\ **Dept. HPER, Northern Michigan University, Marquette, MI, USA
}

\begin{abstract}
Reliability of reactive strength index (RSI) and time to stabilization (TTS) was examined during three maximal effort depth jumps from $30 \mathrm{~cm}(\mathrm{~N}=22)$. Measures of jump height $(\mathrm{JH})$, ground contact time (CT), RSI and TTS were obtained and analyzed for reliability. The $\mathrm{JH}, \mathrm{CT}$ and $\mathrm{RSI}$ were shown to be highly reliable from trial-to-trial $\left(\mathrm{ICC}_{\text {single }}>0.9\right)$. Time to stabilization was not reliable from trial-to-trial $\left(\mathrm{ICC}_{\text {single }}<0.5\right)$. The RSI can be used to monitor performance or to optimize the height of depth jumps. Results suggest that coaches utilizing these procedures with large numbers of athletes may be able to use a single measure of RSI rather than repeated trials. Time to stabilization reliability must be improved before attempting to use it to quantify the landing phase of plyometric exercises.
\end{abstract}

KEY WORDS: plyometrics, balance.

\section{INTRODUCTION:}

Plyometric exercises have been demonstrated to improve power output (Luebbers et al. 2003), agility (Miller et al. 2006) and running economy (Saunders et al. 2006). Depth jumps are one of the most commonly used plyometric exercises and have been frequently used in the literature (Bobbert et al., 1987; Luebbers et al. 2003; Read \& Cisar, 2001). A depth jump requires the athlete to step from a measured drop height and, upon landing on the ground to perform a maximal effort vertical jump, with a short ground contact period. The RSI has been developed as a mechanism to monitor the stress on the musculo-tendinous complex during plyometric exercises such as depth jumps (McClymont \& Hore, 2003) and describes an individual's ability to explosively transition from an eccentric to concentric muscular contraction (Young, 1995). The index can be used to monitor progress in plyometric training or to provide recommendations for optimal depth jump height (McClymont \& Hore, 2003). While the RSI describes the performance of the athlete during the jumping phase of a depth jump, the nature of the landing from such a jump can also be quantified. Time to stabilization (TTS) is a recently developed measure of neuromuscular control that incorporates sensory and mechanical systems to complete the task of a landing after a jump (Wikstrom et al., 2004). The TTS can be calculated by measuring the time taken for vertical ground reaction force to reach and stabilize, within $5 \%$ of the subject's body weight, following the landing from a jump (Wikstrom et al., 2004). The purpose of the current study was to examine the trial-totrial reliability of the RSI and TTS in the jump-landing task of depth jumps. This has not been established in the literature. Establishing the reliability of the RSI would allow coaches to use this variable in the preparation of their athletes with confidence in its reliability. Similarly it would allow researchers to use the RSI to quantify the jumping phase of plyometric exercises. Establishing the trial-to-trial reliability of the TTS measure opens up the possibility for researchers to use this variable to quantify the landing portion of plyometric jumps which has not been extensively examined in the literature.

\section{METHOD:}

Twenty two NCAA Division - I athletes who participated in track and field were recruited to participate in this study. Subjects characteristics were as follows: age (mean \pm S.D.) $20.4 \pm$ 2.4 years, height $175.6 \pm 9.1$ and mass $92.8 \pm 17.2 \mathrm{~kg}$. The University's research ethics committee approved the study and all subjects provided signed informed consent. 
Procedures: Subjects performed a standardized warm-up prior to the collection of data. Following the warm-up subjects performed $3 \mathrm{DJ}$ on a force platform from a height of $30 \mathrm{~cm}$. A one minute rest interval was maintained to ensure sufficient recovery between jumps For the DJ, subjects were instructed to step forward off the box without stepping down, or jumping up and upon contact with the force platform to jump as high as possible and as quickly as possible. For determination of the measure of TTS subjects were instructed to "stick" their landing and to stabilize as quickly as possible, while facing straight ahead and remaining motionless for a period of 7 seconds. Arm position was not controlled throughout the jumping and landing movements as it was desired to keep the plyometric activity as close as possible to that experienced in the training environment. After performing the depth jumps subjects were instructed to stand motionless on the force platform and their body mass was measured. Ground reaction force measurements were obtained for each jump using an AMTI force plate sampling at $1000 \mathrm{~Hz}$. Using the acquired ground reaction force traces, the points of initial ground contact, take-off and landing were identified. Flight time (FT) was calculated as the time between take-off and landing. Jump height $(\mathrm{JH})$ was calculated as $\left(9.81{ }^{*} \mathrm{FT}^{2}\right) / 8$. Ground contact time was defined as the time between the point of initial ground contact and take-off. The reactive strength index (RSI) was calculated as JH divided by CT. Vertical TTS was established as the time from the point of landing to when the vertical force component reached and stayed within $5 \%$ of the subject's body weight for a 1-second duration (see figure 2).

Statistical Analyses: All statistical analysis of the data was carried out in SPSS (C) (Version 13.0). Trial-to-trial reliability analysis of recorded variables used both single $\left(\mathrm{ICC}_{\text {single }}\right)$ and average $\left(I C C_{\text {ave }}\right)$ measures intra-class correlations to absolute agreement. A repeated measures ANOVA was used to determine possible differences between trials. The criterion for significance was set at an alpha level of $p \leq 0.05$. The dependent variables were $\mathrm{JH}, \mathrm{CT}$, RSI and TTS.

\section{RESULTS AND DISCUSSION:}

Table 1 presents the trial-to-trial reliability of all variables measured in the DJ. Jump height and CT were shown to be highly reliable from trial-to-trial $\left(\mathrm{ICC}_{\text {ave }}>0.95, \mathrm{ICC}_{\text {single }}>0.9\right)$. As these two variables constitute the measure of RSI, analysis of this variable also demonstrated high reliability $\left(I C C_{\text {ave }}=0.99, I C C_{\text {single }}=0.97\right)$. Time to stabilization was not reliable from trial-to-trial $\left(\mathrm{ICC}_{\text {ave }}=0.69, \mathrm{ICC}_{\text {single }}=42\right)$

Table 1 Reliability data for all measured variables

\begin{tabular}{lcc}
\hline & \multicolumn{2}{c}{ Intraclass Correlation } \\
& Single measures & Average measures \\
\hline JH & 0.97 & 0.99 \\
CT & 0.93 & 0.98 \\
RSI & 0.97 & 0.99 \\
TTS & 0.42 & 0.69 \\
\hline
\end{tabular}

Figures 1 and 2 display the mean RSI and TTS data for each of the three trials. The group demonstrated a mean RSI of $1.12 \pm 0.49$ across all depth jumps from $30 \mathrm{~cm}$. The mean TTS after landing was $0.97 \pm 0.46 \mathrm{~s}$. The repeated measures ANOVA detected no significant differences between trials 1,2 or 3 for the measure of RSI $(p=0.40), C T(p=0.98), \mathrm{JH}(p=$ $0.25)$ or TTS $(p=0.56)$. 


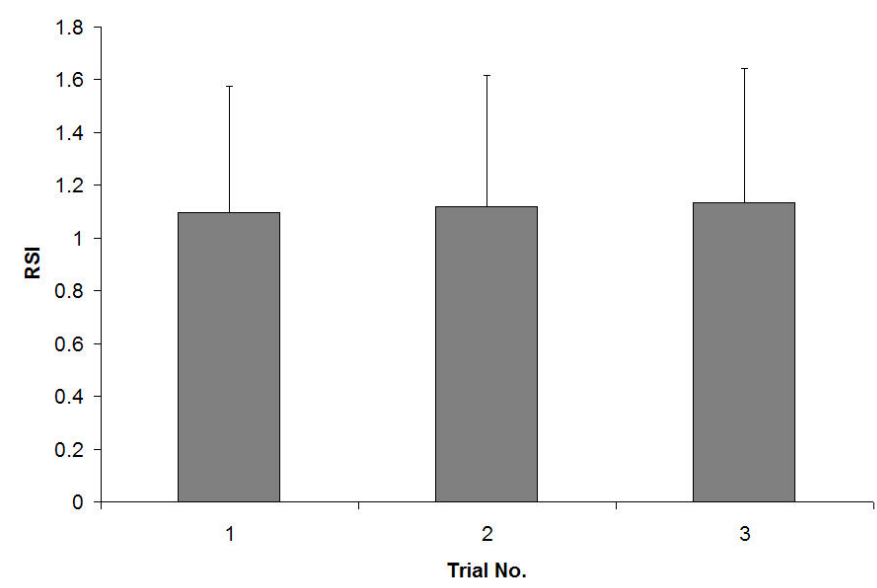

Figure 1: Mean RSI ( \pm S.D.) during DJ. No significant differences observed between trials $(p>0.05)$

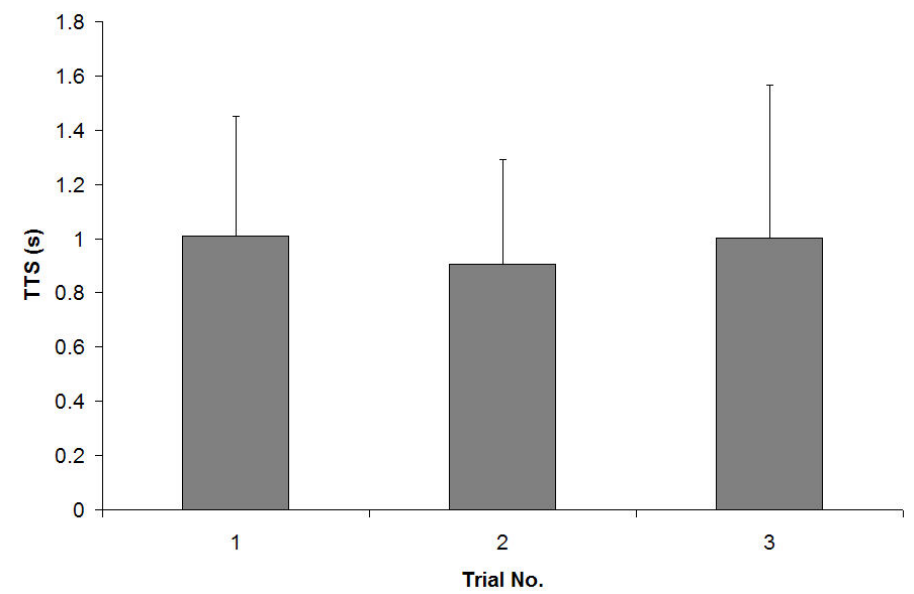

Figure 2: Mean TTS $( \pm$ S.D.) during DJ. No significant differences observed between trials $(p>0.05)$

The reactive strength index was determined to be highly reliable in the DJ. Despite the increasing use of RSI in the practical and research setting, this finding had not previously been established in depth jumps. The intraclass correlations revealed RSI to be highly consistent for single measures. This is an important finding for strength and conditioning coaches or researchers working with large numbers of athletes at a time. Conducting only a single trial from each depth jump height, rather than repeated measures can save significant time and allowed more athletes or subjects to be accommodated. Thus RSI is a quick and convenient tool to use in the practical setting to monitor plyometric progress or to optimize drop heights in depth jump training or in the research setting to quantify the jumping phase of plyometrics.

The mean TTS observed in the present study of $0.97 \pm 0.46 \mathrm{~s}$ is considerably lower than data reported by Wikstrom et al. (2004) of approximately 2.2s. This difference in difficulty of landing is likely due to the protocol of Wikstrom et al. (2004) incorporating a single leg landing while in the current study a two legged landing was used. This finding suggests that the landing task in the present study was easier than that utilized by Wikstrom et al. (2004). Yet, despite this decreased difficulty, poor trial-to-trial reliability was observed in TTS. The major methodological factor that may have negatively influenced the reliability of the measure of TTS is the habituation period given to the subjects in the current study. Subjects were afforded only one practiced repetition of the protocol prior to data collection. Ross et al. (2005) and Wikstrom et al. (2004) both allowed subjects repeated practice trials before beginning the actual experimental trials. Another aspect that may have contributed to the 
lack of reliability observed was that arm position/movement was not controlled throughout the jumping and landing movements. Movement of the upper extremities following a successful landing could disturb ground reaction forces to an extent that might shift the subject outside the $\pm 5 \%$ window of stability despite having landed and balanced successfully.

\section{CONCLUSION:}

This research demonstrated that the RSI, CT and $\mathrm{JH}$ are reliable measures as assessed during depth jumps from $30 \mathrm{~cm}$. The RSI can be used to optimize the height from which depth jumps can be performed or to monitor plyometric training performance. This data suggests that for coaches attempting to conduct this process with large numbers of athletes, it may be sufficient to take just a single measure of RSI from each drop height, rather than repeated trials. Time to stabilization could potentially be a useful tool for strength and conditioning researchers to attempt to quantify the landing phase of plyometric exercises, which has not been thoroughly investigated in the literature. However, the methodological procedures used in the present study produce a measure of TTS which is not reliable amCnd thus renders it unsuitable for use in the research setting. To improve reliability, subjects should be instructed to place their hands on their hips upon landing and be given more practice trails

\section{REFERENCES:}

Bobbert, M.F., P.A. Huijing \& G.J. Van Ingen Schanau. (1987) Drop Jumping I. The influence of jumping technique on the biomechanics of jumping. Medicine and Science in Sports and Exercise, 19, 332-338.

Luebbers, P. E., J.A. Potteiger, M.W. Hulver, J.P. Thyfault, M.J. Carper, \& R.H. Lockwood. (2003) Effects of plyometric training and recovery on vertical jump performance and anaerobic power. Journal of Strength and Conditioning Research, 17, 704-709.

McClymont, D. \& A. Hore. (2003) Use of the reactive strength index as a plyometric monitoring tool. Coaches Information Service. (http://coachesinfo.com/category/rugby/253/)

Miller, M.G., Herniman, J.J., Ricard, M.D., Cheatham, C.C. \& Michael, T.J. (2006) The effects of a 6-week plyometric training program on agility. Journal of Sports Science and Medicine, 5, 459-465.

Read, M.M. \& C. Cisar. (2001) The influence of varied rest interval lengths on depth jump performance. Journal of Strength and Conditioning Research, 15, 279-283.

Ross, S.E., Guskiewicz, K.M. \& Yu, B. (2005) Single-leg jump-landing stabilization times in subjects with functionally unstable ankles. Journal of Athletic Training, 40, 298-304.

Saunders, P.U., Telford, R.D., Pyne, D.B., Peltola, E.M., Cunningham, R.B., Gore, C.J. \& Hawley, J.A. (2006) Short-term plyometric training improved running economy in highly trained middle and long distance runners. Journal of Strength and Conditioning Research, 20, 947-954.

Wikstrom, E.A., Powers, M.E. \& Tillman, M.D. (2004) Dynamic stabilization time after isokinetic and functional fatigue. Journal of Athletic Training, 39, 247-253.

Young, W. (1995) Laboratory strength assessment of athletes. New Studies in Athletics, 10, 88-96. 